\title{
Contrasting and Analyzing Machine and Human Translation: A Case Study on Red Sorghum
}

\author{
Manxin $\operatorname{Lan}^{1 *}$, Linshan Zhao ${ }^{2}$ \\ ${ }^{1}$ The Affiliated High School of Peking University, Beijing 10080, China, ${ }^{*}$ Corresponding author. \\ manxinlan@gmail.com \\ ${ }^{2}$ The International Communication Center of Hengshui No.1 High School, Hengshui 053000, China
}

\begin{abstract}
The quality of machine translation is an important part in machine language competence. Currently, there are still many differences between machine translation and human translation. The quality of machine translation still could not reach the quality of human translation. We take a Chinese literary text, Mo Yan's Red Sorghum, as the material to translate, and used LIWC2015 to analyze the differences between machine and human translation. Also, we make a linguistical attribution analysis to find out the reasons behind the errors machines make. For the engines of machine translation, we choose Baidu, Youdao and Google as machine translate engines. By analyzing the results from LIWC2015 and error analysis, we find that compared to human translators, machine translate engines tend to use simpler words and often fail to recognize discoursal connection; the errors machine translate engines make are usually caused by long sentences, Chinese names, encyclopedic knowledge, unique expressions and constructions in Chinese and literary expression.
\end{abstract}

Keywords: Machine translation, Error analysis, Chinese-English translation, Literary text

\section{INTRODUCTION}

Machine language competence is the competence of machine to process language intelligently [1]. Machine translation is one of the techniques that require high machine language competence. However, there are limitations of machine translation: the quality of translation output is lower than human translation, and they make mistakes. As neurolinguistics thrived in academia [2], it is claimed that enabling the machines to imitate the way human brain process language might be a good way to improve machine language competence, and therefore improve the quality of machine translation [1]. Before doing so, knowing current limitations of machine translate is important. Current studies have shown many examples of analyzing errors in machine translation [3-6] and how to evaluate their qualities [7,8]. However, only few has considered the issue from the perspective of machine language competence.

In this study, we use the software LIWC2015 that includes approximately 90 output variables to analyze and compare the translation results from three translate engines, Baidu, Youdao, Google and human translation. We have chosen the variables of word count, words $>6$ letters, dictionary words, and pronoun [9]. Then, we categorize the errors in machine translation by using linguistical attribution analysis. Finally, we replicated the errors in this way, we could explore the reason why they make such errors.

The text we chose to translate is from the Red Sorghum, a novel by Mo Yan, a Chinese Nobel Prize winner. The narrator tells the story of his family's struggles during a war. The reason why we choose a novel is that recent work shows that machine translate engines could handle illustrative text well [10], so in order to find limitations of them, we chose a text that is a literary narrative. Also, since Mo Yan has won the Nobel Prize, he is internationally well-known. Thus, the quality of human translation is guaranteed.

\section{DIFFERENCES BETWEEN MACHINE TRANSLATION AND HUMAN TRANSLATION}

In this study we used the LIWC2015 to analyze the text translated by machines and compared them with the results of human translation. Among about 90 output variables provided by LIWC2015, we chose word count, words $>6$ letters, dictionary words, total pronoun, $1 \mathrm{st}$ person singular, 1st person plural, 3rd person plural and articles. The results of these variables showed significant 
difference between machine and human translation. Except for word count, all of the data of the variables indicate the percentage of the words included in the variables in the text. Here are the results:

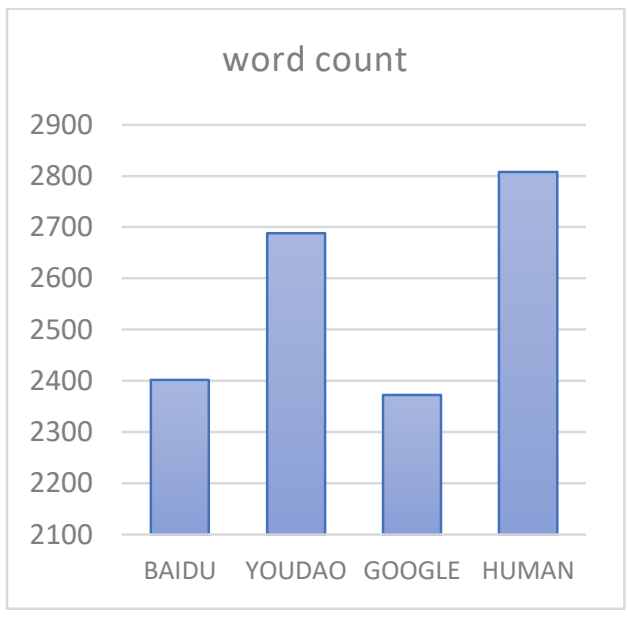

Figure 1 word count

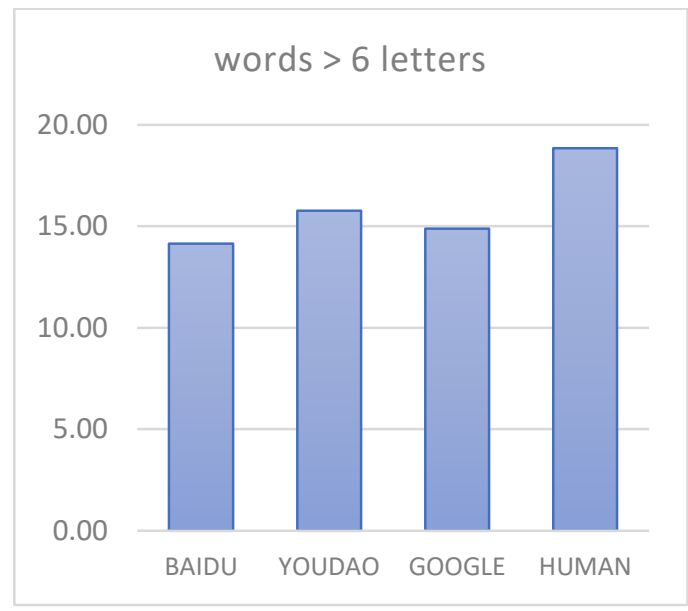

Figure 2 proportion of words $>6$ letters

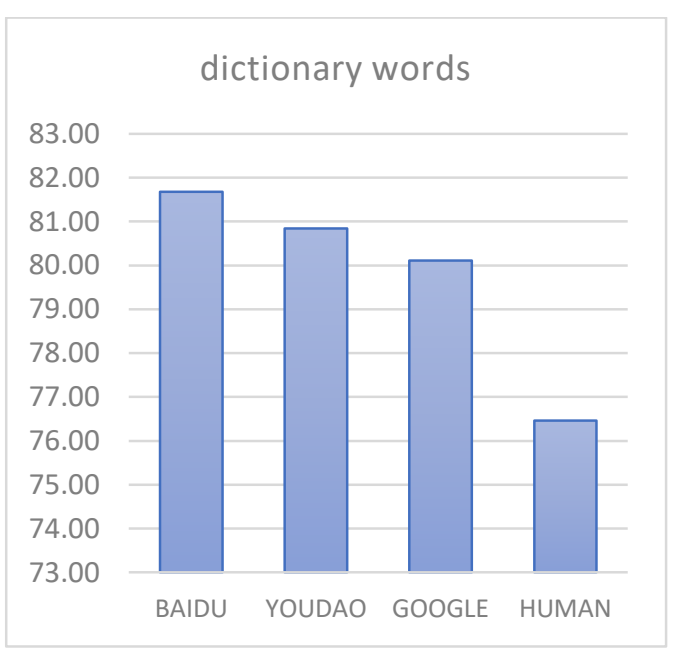

Figure 3 proportion of dictionary words

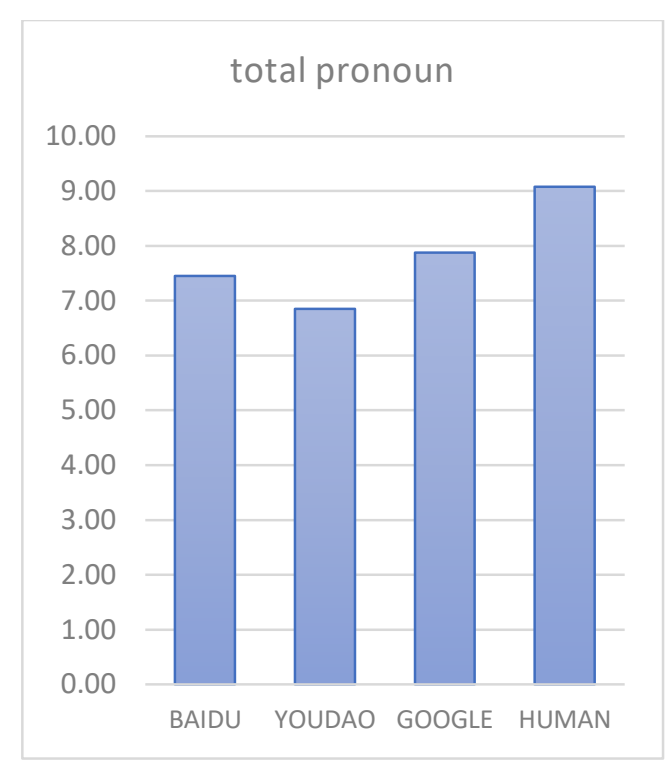

Figure 4 proportion of total pronoun

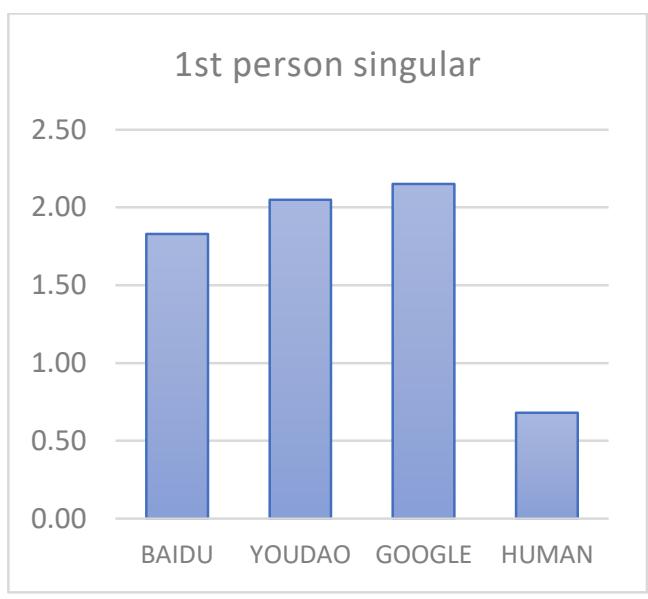

Figure 5 proportion of 1 st person singular

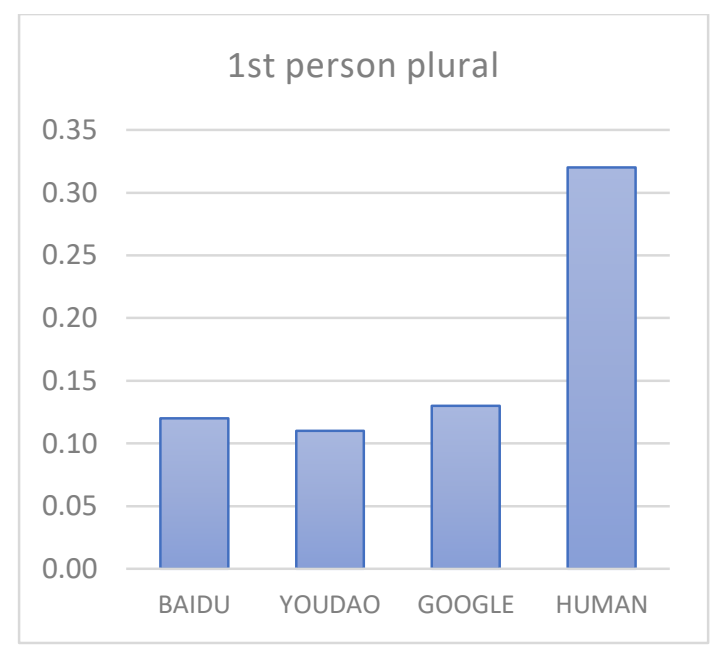

Figure 6 proportion of 1st person plural 


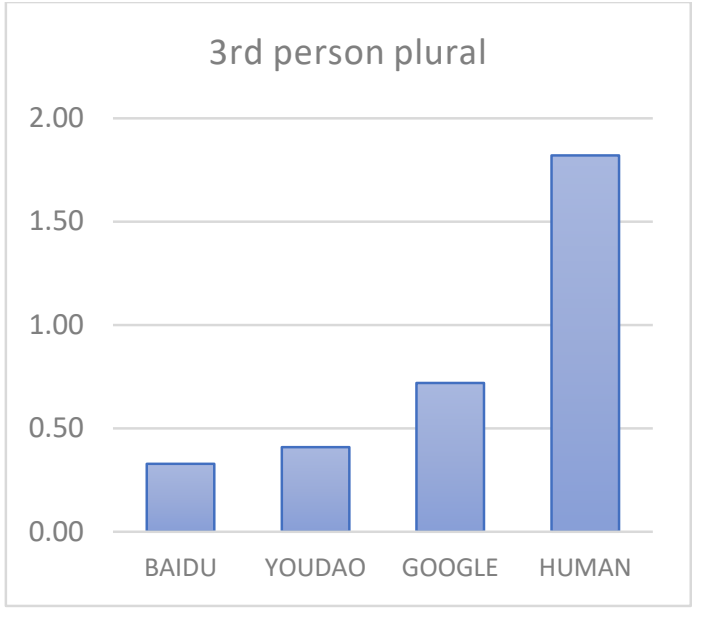

Figure 7 proportion of 3rd person plural

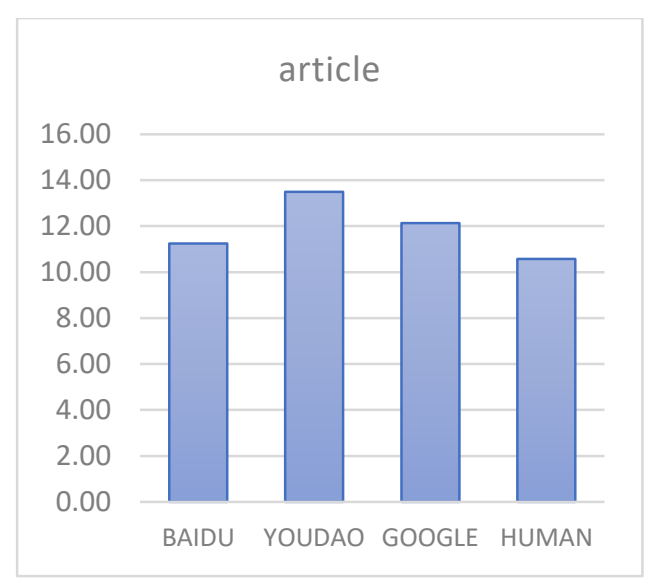

Figure 8 proportion of article

From the results of the variables words $>6$ letters and dictionary words, it is reasonable to infer that machine translation tends to use simpler words than human translation. Words that include more than six letters usually are not common words that is widely used in daily life. Also, in the results of dictionary words, machine translation tends to use more dictionary words. This indicates that compared to human translation, machine translation uses less words that are not in the dictionary of LIWC2015. Thus, with the results of words $>6$ letters and dictionary words, we find that even though machine translate engines would not have limited word bank compared to human translators, machine translate engines would choose simpler words.

The reason behind this phenomenon might be complex. The first possible reason might be that machines have trouble interpreting the original text. They might not interpret the meaning of the original word comprehensively, and thus would choose a word that machines believe would convey the same meaning: a simpler word. In short, machines interpret the original text as a simple meaning, so the text they come out with have simpler words. This also explains why the word count in machine translation is more than human translation. Machines do not understand the complex meaning, and so would just express in a simpler way, resulting in a shorter text.

Another possible reason for this might be that the machine translate engines are designed to output simpler words. If machine translate engines output results that include many complex words, users might be confused and would not interpret the original text, which is opposed to their purpose of using machine translate engines.

From the results of the pronoun and article, we could infer that machine sometimes fail to recognize connections in the text, that is, they could not interpret discoursal meaning. The main purpose of using pronouns and articles is to mention the object that is already mentioned in previous texts. Thus, a difference in the use of pronouns and articles between machine and human indicates that machine have trouble connecting previous texts when translating. Here is an example of different pronoun and article:

Original text: [我的]父亲五岁时......

Human: At the age of five, he [my father] ...

Baidu: When his father was five...

Youdao: When my father was five years old...

Google: When the father is five years old...

From this example, we could see that only human translation used the word "he", while all of the three machines translate 父亲 (which means father in English) in "(an adjectival possessive pronoun / an article) father". It is reasonable for human translator to use the word "he" because preceding sentences has already mentioned this character. However, the machine translations instead chose to include the word "father". The reason behind this is that the machines do not notice the relation in the text, but only translate sentence by sentence, neglecting the perspective of seeing the discourse as a whole. Also, in this example, only Google translate used an article in front of the word father. Youdao put "my" in front of "father", and Baidu put "his" in front of "father", which is incorrect. From the context in the original text, we could see the word 父亲 refers to my father. However, Baidu translate did not interpret it correctly. This indicates that Baidu failed to recognize the connection between this sentence and the preceding text.

\section{ANALYSIS}

\subsection{Long Sentences}

Long sentences are prominent in Chinese language. In English, clauses or phrases in a sentence are coordinated with or subordinated to one another syntactically while in Chinese they are placed one after another without coordinating connectives. That makes translating a long 
sentence difficult. Different "parts" of a sentence are diffusely connected to each other, so when translated into English, there will be omissions in terms of prepositions and other small particles. Here are two examples:

Original text: 他跟着后来名满天下的传奇英雄余 占鳌司令的队伍去胶平公路伏击日本人的汽车队。

Human translation: My father was joining the forces of Commander Yu Zhan'ao, a man destined to become a legendary hero, to ambush a Japanese convoy on the Jiao-Ping highway.

Baidu: He followed the legendary hero commander $Y u$ Zhanao's team to the Jiaoping highway to ambush the Japanese car team.

Youdao: He followed the legendary hero Yu Zhan 'ao commander's team to the Jiaoping road to ambush the Japanese motorcade.

Google: He followed the team of the legendary hero, Commander Yu Zhanao, who would later become famous all over the world, to ambush the Japanese car team on Jiaoping Highway.

In this example, one part that is omitted is the word 后来, which means “eventually" here. Also, the machine translations have minor grammar errors here and there. The reason why the machines make this kind of error is that when confronting long sentences, machines have difficulties in analyzing the structure because there are too many elements in the sentence.

When we replicated the error, we shortened the original sentence above to see what makes the sentence too long to translate.

Revised original text 1: 他跟着后来名满天下的传 奇英雄余占鳌司令的队伍伏击日本人的汽车队。

Human: My father was joining the forces of Commander Yu Zhan'ao, a man destined to become a legendary hero, to ambush a Japanese convoy.

Baidu: He followed the team of commander $\mathrm{Yu}$ Zhanao, a legendary hero, to ambush the Japanese car team.

Youdao: He followed the legendary hero, Commander Yu Zhanao, who ambushed the Japanese motorcade.

Google: He followed the team of the legendary hero, Commander Yu Zhanao, who became famous all over the world, to ambush the Japanese car team.

Revised original text 2: 他跟着后来名满天下的余 占鳌司令的队伍去胶平公路伏击日本人的汽车队。

Human: My father was joining the forces of Commander Yu Zhan'ao, a man destined to become famous, to ambush a Japanese convoy on the Jiao-Ping highway.

Baidu: He followed the later world-famous Commander Yu Zhan 'ao's team ambushed the Japanese motorcade.

Youdao: He followed the later world-famous commander Yu Zhan 'ao's team to Jiaoping road ambush the Japanese motorcade.

Google: He followed the team of Commander $\mathrm{Yu}$ Zhanao, who became famous all over the world, to ambush the Japanese car team.

In revised version 1, we deleted the phrase 去胶平公 路 (on the Jiao-Ping highway). However, only Google translated the sentence correctly. Baidu and Youdao still omitted the phrase 后来名满天下的 (destined to be famous all over the world). In revised version 2, we deleted the phrase 传奇英雄 (legendary hero). This time, all of the three translate engines translate the sentence correctly. Through replicating the error, it could be inferred that the reason why machines would make errors when confronting long sentences is that the structure of sentence is too complex. In the case above, there are too many attributives in front of 余占鳌司令, thus the machines would be confused, resulting in omitting some of the attributives.

\subsection{Unique Expression in Chinese}

We also found that machines have trouble translating unique expressions in Chinese, which include slang, idiom, and dialect. Here is an example:

Original text: 父老乡亲

Human: the people of my father's generation

Baidu: my parents and villagers

Youdao: the people of my father's generation

Google: my fathers and villagers

Original text: 老伙计

Human: foreman

Baidu: old man

Youdao: old friend

Google: old man

In the first example, 父老乡亲 is an idiom. Both Baidu and Google translate the word directly, ignoring the actual meaning of the word: 父老 could be directly translated to my parents or my parents, and 乡亲 could be directly translated to villagers. In the second example, 老伙计 (foreman) is a slang. However, the three machines fail to recognize this but instead translated it 
literally. 老 could be translated to old, and 伙计 could be translated to man or friend.

From above, we could infer that machine translate engines have trouble translating unique Chinese expressions. The reason might be that they do not have the word in their dictionaries. When they confront this kind of words, they would divide the word into parts and translate directly part by part.

\subsection{Construction}

Chinese language is vivid and often uses symbolic or figurative expressions to get to the point. In the source text, such constructions are prominent. A direct translation of these expressions will get the wrong meaning that the author meant to express. However, machine translations often translate things bluntly, so this kind of mistake is frequent. Here are some examples:

Original text: 一老一小

Human: the two of them, one old and one young

Baidu: as soon as I was old and small

Youdao: one old and one small

Google: An old one is a small light

一老一小 has the construction of - $\mathrm{A}$ - $\mathrm{B}$, where $\mathrm{A}$ and $\mathrm{B}$ each represents a single Chinese character. If they combine together, an idiom of some kind is formed. You cannot take out the single characters and translate them separately and directly, like in the machine translations. Here, the character - loses its literal meaning of the number "one". Instead, the word should be taken as a whole and translate into "two people, one of them old and the other young".

\section{Original text: 羊粪稀稀拉拉像震落的黑豆}

Human: scattered goat pellets like little black beans

Baidu: Sheep dung is as thin as black beans

Youdao: Sheep droppings scattered like black beans

Google: The sheep dung is sparsely like black beans shaking down

The word 稀稀拉拉 could be categorize as the construction of $\mathrm{AABB}$. This kind of construction is unlike ABAC: it cannot be divided. Instead, this kind of word can only be interpreted as a whole. In 稀稀拉拉's case, both 稀稀 and 拉拉 have no real meaning. However, the machines translate this word in that way, so it becomes "dilute lala" which makes no sense. The actual translation should be "scattered".

In order to find the reason why machines could not translate words that have certain constructions, we tried to replicate the error.
First we put the word 稀稀拉拉 in another sentence:

Original text: 人们稀稀拉拉地跟着她走出去。

Human: People followed her out in a sparse way.

Baidu: People followed her out in a sparse way.

Youdao: The people followed her thinly.

Google: People followed her out sparsely

We could see that all of the three translate engines translate the sentence correctly. However, there is a major difference between the sentence we created and the sentence from Red Sorghum. In the sentence we created, there is a 地 after the word 稀稀拉拉, which indicates that 稀稀拉拉 here is an adverbial. Thus, the reason why the machines could not translate the sentence from Red Sorghum correctly is that there are no indicators to indicate the function of the word. Thus, we add a 的 after 稀稀拉拉 in the original sentence to indicate 稀稀 拉拉 is an attributive:

Revised original text: 羊粪稀稀拉拉的像震落的黑 豆

Human: scattered goat pellets like little black beans

Baidu: Sheep dung is as thin as black beans

Youdao: Sheep droppings scattered like black beans

Google: The sheep dung is sparsely like black beans shaking down

This time, all of the translate engines translate correctly. Therefore, we could conclude that the reason why machines fail to translate words with certain constructions is that these words do not have fixed function in the sentence. Only when the original text have a functional word like 的 and 地, they could translate correctly.

\subsection{Literary Expression}

From the results of machine translation, we also find some errors caused by the literary expressions in the original text. Here are two examples:

Original text: 一队队暗红色的人在高梁棵子里穿 梭拉网，几十年如一日。

Human: Over decades that seem but a moment in time, lines of scarlet figures shuttled among the sorghum stalks to weave a vast human tapestry.

Baidu: Teams of dark red people have been shuttling through the sorghum trees for decades.

Youdao: Teams of dark red people in the sorghum in the back and forth nets, as a day for decades. 
Google: Teams of dark red people shuttled through the sorghum trees to pull the net, like a day for decades.

Original text: 高粱高密辉煌，高粱凄婉可人，高 粱爱情激荡。

Human: Tall and dense, it reeked of glory; cold and graceful, it promised enchantment; passionate and loving, it was tumultuous.

Baidu: Sorghum Gaomi is brilliant, sorghum is sad and pleasant, and sorghum love is exciting.

Youdao: Sorghum Gaomi brilliant, sorghum wan but people, sorghum love agitate.

Google: Sorghum high density is brilliant, sorghum is sad and pleasant, and sorghum love is surging.

In the first example, the phrase “暗红色的人” is translated to "dark red people" by all of the three translate engines, while the phrase is translated to "scarlet figures" by human translator. Dark red figure is a direct translation which is not necessarily wrong. However, compared to "scarlet figures", it fails to convey the original meaning of “暗红色的人”. This is because the character “人” in Chinese could be translated in to both "people" and "figure". However, in the original text, “人 " conveys a more abstract meaning, thus the word "figure" would be more appropriate. The reason why machines make this mistake is that the original phrase " 暗红色的人” is not commonly used in daily life. In other words, it is a temporary combination of words in a literary context.

In the second example, there are two major errors we want to discuss here. The first one is about the word “高 密”. In human translation, this word is translated into "tall and dense". However, in Baidu and Youdao, it is transliterated into Gaomi. The word 高密 is not a word in dictionary. Instead, it is a word that is combined with two characters 高 and 密. The two characters mean "tall" and "dense" respectively. The author combined these two characters for the sake of literary effect and rhythm in the sentence. Thus, the word 高密 is a temporary usage in literary context. The reason why the machine translation engines make error is that they could not interpret the word as a combination of two words, but only get confused about an unfamiliar word combination.

The second error in the second example is about the word “凄婉可人”. Human translator translated the word into "cold and graceful", while Baidu and Google all translated it into "sad and pleasant". Youdao translated it into "wan but people". "Wan" is the pronunciation of the character 婉, and "but people" is the direct translation of “可人”. The word 凄婉可人 is also not an dictionary word in Chinese. Thus, the machines make the error also because of temporary usage in literary context.
When we replicate this kind of error, we chose a sentence from Shi Tiesheng's "I and the Temple of Earth", which is also a literary text. Here is the original text, the result of three machine translation engines and human translation:

Original text: 当它熄灭着走下山去收尽苍凉残照 之际，正是它在另一面燃烧着爬上山巅布散烈烈朝 晖之时。

Human: While it is stepping downhill to collect the desolate fading light, it is precisely at that moment that it is burning splendidly as it climbs to the summit from the other side.

Baidu: When it went down the mountain to collect the bleak afterglow, it was just when it was burning on the other side and climbing to the top of the mountain to spread the bright sunlight.

Youdao: When it went down the mountain to extinguish the desolation, it was burning on the other side of the mountain to climb the sun.

Google: When it extinguished and walked down the mountain to collect the desolate afterglow, it was when it burned on the other side and climbed to the top of the mountain to spread the fierce sunrise.

In the original text, 残照 is not a dictionary word in Chinese. It is also a temporary usage that the author use for the sake of literary effect and rhythm, just like the 高 密 (tall and dense) and 凄婉可人 (cold and graceful). Human translator translated the word into "fading light". Baidu and Google both translated the word into "afterglow", which is acceptable. However, Youdao omitted the word and just translate the phrase 苍凉残照 (desolate fading light) into desolation, which is not acceptable.

By replicating the error, we found that it is difficult for machines to translate temporary usage of words in literary context. The reason might be machines are not flexible when confronting new combinations of words. When they confront this kind combination, they sometimes transliterate is or omit it.

\section{CONCLUSION}

Machine translation is an important field that requires machine language competence. In this study, we find that comparing to human translator, machine translate engines tend to use simpler words and have trouble noticing discoursal connections. We attribute the errors they make into four reasons: long sentences, unique expressions in Chinese, construction and literary expressions. We could see that there is still limitation in machine translation engines especially when they are translating literary texts. Still, the machine language competences between three translate engines are different: Google translate does 
better when translating long sentences and literary expressions, which indicates that its competence of processing sentence structure is higher; Youdao translate does better when translating unique expressions in Chinese, which might be attribute to its Chinese-Internetbased data base.

Machine language competence in machine translate engines needs to be improved. The most effective way might be changing their inner structure: to enable machine translation to reach the quality of human translation, they must process language they way human brain process language.

\section{ACKNOWLEDGMENT}

We would like to extend our sincere gratitude to our instructor, Prof. Andrew Nevins and our teaching assistant Yi Zhang for their instructive advice and useful suggestions on our paper. We also want to extend our gratitude to Yuxiao Wang who helped us analyzing the texts.

\section{REFERENCES}

[1] Geng, Libo et al. 2014. "The Research on Machine Language Competence: Status Quo and Prospects”. Language Science (January), pp. 34-41

[2] Yang, Yiming. 2007. "Theoretical Hypotheses on Language and Their Neurological Basis". Language Science (March), pp. 60-83

[3] Kai, Bao. 2017. "A Preliminary Study on Error Types and Correction Methods of Google Translation". Chinese Science and Technology Translators Journal (November), pp. 20-23

[4] Costa, Ângela et al. 2015. “A Linguistically Motivated Taxonomy for Machine Translation Error Analysis”. Machine Translation (June), pp. 127-161

[5] Jesus Martins, Débora Beatriz de and Medeiros Caseli, Helena de i. 2015. "Automatic Machine Translation Error Identification". Machine Translation (March), pp. 1-24.

[6] Li, Rongrong. 2017. "Research on C-E Online Machine Translation - Case Study and Analysis on Three Online MT Tools". Nanchang, Nanchang University

[7] Wang, Jinshuan and Xin, Wan and Dong, Ziyun. 2018. "Translation Quality Evaluation Methods and Their Applications in Computer Translation Evaluation Systems". Chinese Translators Journal (NO.4), pp. 73-78

[8] Williams, M. 2004. "Translation Quality Assessment: An Argumentation-centered Approach". Ottawa: University of Ottawa Press
[9] Pennebaker, J.W. and Boyd, R.L. Jordan, K., and Blackburn, K. (2015). "The Development and Psychometric Properties of LIWC2015”. Austin, TX: University of Texas at Austin

[10] Yang, Yanling and Zhu, Anbo. 2018. "Research on the Applicability of Online Translation to Poetry Translation: A Case Study on "The Daffodils". Translate Forum (March), pp.43-48 